enlargement of the wound by which they are accompanied, but elevate the intruded substance with a pair of fine forceps, and excise its investing membrane. The conjunctiva may be freely incised and excised, without injury to the eye, of which we had abundant proof during "the squinting mania."

[To be continued.]

\section{CASE OF ANEURISM OF THE THORACIC AORTA}

BURSTING INTO THE TRACHEA, SIMULATING CHRONIC LARYNGITIS.

By WILliam HENRY GOOCH, M.D., Edin., PHYSICIAN TO THE KENT AND CANTERBURY HOSPIFAL.

The subjoined case was read to the East Kent and Canterbury Medical Society, and is an instructive example of aneurism of the arch of the aorta, which was not discovered during life, but produced symptoms resembling those of chronic laryngitis.

A carpenter, married, aged 44, of strumous habit, middle sized, and temperate, was admitted Sept. 20th, 1850, into the Kent and Canterbury Hospital. It appeared that about a twelvemonth previously he fell from the top of a house, and soon afterwards spit a little blood; the hæmorrhage, however, did not return. Four months afterwards he began to complain of a sense of fulness and tenderness about the upper part of the windpipe, which he attributed to a neglected cold, as he coughed a little at the time. Nothing was done for the relief of these symptoms for three months. He was then blistered, the medical attendant regarding his complaint as inflammation of the chest. No relief ensuing, he sought admission into the hospital. He was then reserved and dejected, and much wasted. He complained chiefly of a sense of obstruction behind the thyroid cartilage, with tenderness and soreness in that situation when he spoke or coughed. The cough was hoarse, and often shrill, and at the end of each fit of coughing the breathing became extremely hurried, especially at night, so that he could sleep but little. His voice was hoarse, and sometimes whispering, and he expectorated a scanty frothy mucus.

Auscultation disclosed a feeble respiratory murmur throughout the left lung, particularly at its apex, without rhonchus. The rocal resonance was morididly loud, and the sound on percussion dull anteriorly and posteriorly. In the right lung, with the exception of a little sibilous rhonchus in the upper lobe, the sounds heard by the stethoscope, and percussion, were natural. The systole of the heart was feeble, but the sounds and rhythm were healthy. No unnatural arterial pulsation was discovered anywhere. The pulse was 84 , soft, and weak. The appetite was impaired, but the tongue looked clean.

Considerable relief was afforded by cupping in the inter-scapular region, by counter-irritation on each side of the larynx, and by mercurials, with expectorants and sedatives. Dyspnca was alleviated, and sleep secured, by a nightly dose of chloroform. Slight œdema of the ankles now appeared, but with that exception the patient had improved and gained strength, from a nutritious diet and cod-liver oil, when soon after midnight of the 23rd of November he was seized with a sudden and very violent fit of coughing, during which an enormous quantity of blood rushed from his mouth, and he was dead in an instant. A slight aggravation of the cough had been noticed for a few days before the awful event.

Post-mortem examination on the second day after death.-The larynx and trachea showed no marks of disease. The right lung was emphysematous, and did not collapse when the chest was opened. The lung at its upper part was adherent to the costal pleura by long bands of lymph, both anteriorly and posteriorly. Several large red patches were scattered over the surface of the middle and lower lobe, which condition was thought to depend on the entrance of blood into the minute divisions of the air tubes, several small coagula being found in the larger bronchi. The left lung was universally adherent by a thick firm layer of lymph, and did not crepitate, the whole of its tissue from apex to base being in a state of grey hepatization. Three or four cretaceous deposits were found, of the size of half a grey pea, on making sections of the lung. The bronchial ramifications appeared dilated, and their lining membrane thickened, and intensely injected. The pericardium contained the usual quantity of fluid, and presented some white fibrinous patches on the surface of the right ventricle, together with adhesions between the surfaces at the base, around the great vessels. The heart was somewhat large, but its cavities and valves were natural. The aorta presented in its first portion patches of white thickening of the inner coat, and atheromatous deposits, and at more than one spot a circumscribed superficial dilatation of the coats had taken place. Immediately beyond the left subclavian artery there appeared a large opening, that would readily admit the fore-finger, which communicated with an aneurism, of the size of a small orange; it was situated between the aorta and trachea, and contained a small loose mass of fibrin, but had no concentric laminæ. The rings of the lower part of the trachea were bare in the posterior wall of the sac, for a space equal to the circumference of a sixpence. At this spot there were two distinct openings that passed into the trachea at its bifurcation, one of these, more properly speaking, led into the commencement of the right bronchus. The margins of these openings on the tracheal side were surrounded with tufts of lymph, adhering pretty firmly. The left recurrent nerve, after passing round the corresponding bronchus, became embedded in the wall of the aneurism. The glands between the bronchi were much enlarged, and filled with a hard cretaceous substance.

In reflecting on the case now detailed, one cannot fail to be struck with the undoubted relief afforded by the treatment to the more urgent symptoms, at the 
same time that the aneurismal disease must be considered to have been advancing. To the congested and hepatized condition of the lungs diagnosticated during life, and discovered after death, we may point as satisfactorily explaining this apparent anomaly.

But the great practical lesson which the case suggests has reference to diagnosis; fresh proof is afforded by it of the obscurity which surrounds the progress of aneurisms about the commencement of the aortic arch. In the majority of instances the symptoms that result from compression of the surrounding organs, offer only equivocal evidence; it remains for a pulsating tumour, or a sudden arterial hæmorrhage, to reveal the true nature of the affection. Sometimes, indeed, the trachea, the œsophagus, or the recurrent nerve, sustain such impediments to their functions from pressure, that the diagnosis is not a matter of difficulty. On the other hand may be cited, as a striking contrast, the case recorded by Mr. Lawrence in the "Medico-Chirurgical Transactions," Vol. 6, where an aneurism of the arch of the aorta so pressed on the trachea as to ulcerate its lining membrane, and yet the patient did not experience any dyspnœa, death arose from a different cause, and the discovery of the aneurism, which was small and filled with firm laminated coagula, was quite accidental.

Laennec, when speaking of aneurism of the thoracic aorta, remarks :- "It cannot be known with certainty till it shows itself externally; it can hardly be suspected even when it compresses some important organ and greatly deranges its functions."

And in support of the same doctrine, the case of the late Mr. Liston may be adduced. At first, it will be remembered, his symptoms were attributed to tubercular condensation of the superior lobe of the lungs. It was thought doubtful whether the dyspnœa depended on enlargement of the bronchial glands, or on aneurism; the latter was only suspected by the distinguished physicians in attendance. This suspicion proved to be correct, for a false aneurism of the aorta was found of the size of an orange, which had burst into the trachea, and, of course, had led to the copious hæmoptysis that took place five months before death.

In the Catalogues of the Museum of the Royal College of Surgeons, and of St. Bartholomew's Hospital, three cases of aneurism pressing on the trachea are described, in two of which an opening was made into the trachea to relieve the symptoms*

* The first (No. 1665, in Catalogue College of Surgeons Museum, London,) is described as aneurism of the posterior and upper part of the arch of the aorta, attached by a narrow neck, and opening into it by an oval mouth, half an inch wide, immediately below the origln of the innominata. The sac pressed on the trachea and burst into its front part just above the bifurcation, by a small irregular opening. Laryngotomy had been performed (probably for symptoms pointing to the larynx.)

The second case (Vol. 1, series 13, No. 69, Catalogue St. Bartholomew's Hospital Museum, London,) is described as aneurism of the upper part of the arch of the aorta, involving the arteria innominata, slightly com. pressing the trachea. The patient was supposed to have chronic asthma; to relieve suffocation the trachea was opened. In the operation a large thyroid vein was opened, and the patient died.

The third case (Vol 1, series 13, No. 18, same Catalogue,) is that of aneurism of the arteria innominata pressing on the trachea. The patient was twenty years old, and subject to fits of dyspncea, and in one of these she died. The canal of the trachea is slightly narrowed by the pressure of the innominata.
In the instance under consideration, the absence of all physical signs indicative of disease of the heart or its large vessels, the evidence of solidification in the left lung, the peculiar character of the voice and cough, with other symptoms, led to the inference that the chief lesion was strumous laryngitis, probably advanced to the ulcerative stage, (with pre-existent pneumonia.)

Post-mortem examination corrected the error by dis. covering another and an adequate cause of the affection of the throat, in the fact of the recurrent nerve being involved in the aneurismal sac; while the condition of the left lung, and the position of the aneurism, explained the paroxysms of difficult breathing.

It is right to add that a suspicion of some unusual pressure upon the recurrent nerve, like that whish would be caused by enlarged bronchial glands, had been expressed by me shortly before the patient's decease.

\section{ON THE VARIETIES OF CRANIAL PRESENTATION.}

\section{BY JOSEPH GRIFFITHS SWAYNE, M.D., LoND.,} Lecturer on Obstetric Medicine at the Bristol Medical School.

The relative frequency of the different cranial presentations has long been a vexata questio between the professors of the obstetric art in France and Germany. The French accoucheurs have mostly taken one side of the question, and the Germans the opposite : whilst the greater number of our own countrymen have been disposed to adopt the views of the latter. As the point at issue is one which is of some importance, and which even the limited experience of individuals may tend in some degree to settle, I am induced to bring forward the particulars of a certain number of cases, which have been carefully noted for the purpose. In so doing I have availed myself only of those which have fallen under my own immediate observation, for the most part in private practice; and have refrained from making use of a considerable number which have been attended by the pupils of the Bristol Medical School, under my superintendence, because the descrimination of the varieties of cranial presentation usually requires a higher degree of the " tactus eruditus," than can reasonably be expected in those who are yet in statu pupillari.

The cases I am about to bring forward have not been selected, but have been taken as they occurred, and amount in all to 286 . In classifying these according to the presentation, I shall only refer to the four kinds of cranial presentation which are commonly recognised amongst obstetric authors. In the first of these, which is the ordinary presentation of the head, the occiput of the child is turned towards the left acetabulum of the mother's pelvis; in the second, it is towards the right acetabulum; in the third, towards the right sacro-iliac synchondrosis ; and in the fourth, towards the left sacroiliac synchondrosis. The two first of these positions have been called by Velpeau and some of the French authors, the occipito-anterior positions, because in them 